\title{
Ultralow-energy magnon anomaly in yttrium iron garnet
}

\author{
Shin-ichi Shamoto $\odot,{ }^{1,2,3, *}$ Yukio Yasui, ${ }^{4}$ Masato Matsuura $\odot,{ }^{2}$ Mitsuhiro Akatsu $\odot,{ }^{5}$ Yoshiaki Kobayashi, ${ }^{6}$ Yuichi Nemoto, \\ and Jun'ichi Ieda $\otimes^{1}$ \\ ${ }^{1}$ Advanced Science Research Center, Japan Atomic Energy Agency (JAEA), Tokai, Ibaraki 319-1195, Japan \\ ${ }^{2}$ Neutron Science and Technology Center, Comprehensive Research Organization for Science and Society (CROSS), \\ Tokai, Ibaraki 319-1106, Japan \\ ${ }^{3}$ Advanced Meson Science Laboratory, RIKEN, Wako, Saitama 351-0198, Japan \\ ${ }^{4}$ School of Science and Technology, Meiji University, Kawasaki, Kanagawa 214-8571, Japan \\ ${ }^{5}$ Department of Physics, Niigata University, Niigata, Niigata 950-2181, Japan \\ ${ }^{6}$ Department of Physics, Graduate School of Science, Nagoya University, Nagoya 464-8602, Japan \\ ${ }^{7}$ Graduate School of Science and Technology, Niigata University, Niigata, Niigata 950-2181, Japan
}

(Received 16 April 2020; revised 19 June 2020; accepted 20 July 2020; published 11 August 2020)

\begin{abstract}
Ultralow-lying magnon energy spectrum in yttrium iron garnet (YIG) has been studied by inelastic neutron scattering in an energy range from 10 to $45 \mu \mathrm{eV}$. When a magnetic field of approximately $0.1 \mathrm{~T}$ was applied along [111] direction, ultralow-energy magnon anomaly was found at $10 \mathrm{~K}$, suggesting the closure of the Zeeman energy gap. The anomaly was also observed in the temperature dependence of magnetization under a magnetic field along the [111] direction below $30 \mathrm{~K}$. The specific heat capacity confirms the closure of the Zeeman energy gap at the magnetic field direction. All these anomalies strongly support the magnetic crossover below $30 \mathrm{~K}$ under the magnetic field along [111], in addition to a precursor anomaly below $150 \mathrm{~K}$.
\end{abstract}

DOI: 10.1103/PhysRevResearch.2.033235

\section{INTRODUCTION}

Yttrium iron garnet (YIG) with a composition of $\mathrm{Y}_{3} \mathrm{Fe}_{5} \mathrm{O}_{12}$ is a well-known ferrimagnet used for various industrial applications. It is also commonly used for spintronics [1-4] and magnonics [5,6] due to the long magnon lifetime observed as a narrow linewidth of ferrimagnetic resonance [7]. The magnons in this insulating oxide carry the spin current without charge current flows and resulting Joule heating loss. Hence, YIG with a weak magnetic anisotropy and long lifetime is indispensable for spintronic devices [8]. Especially, the weak magnetic anisotropy becomes important for the spin current, because in addition to the thermally excited magnons, the subthermal low-lying magnons prominently contribute to the spin current generation [9]. Any gap in the magnon dispersion reduces those magnons, decreasing the spin current. Therefore, understanding the ultralow-energy magnons is important to study the intrinsic mechanisms of the spintronic effects such as the spin Seebeck effect [2-4].

So far, the spin Seebeck effect is well explained by a magnon density of state (MDOS) model below $30 \mathrm{~K}[8,9]$. The deviation from the model above $30 \mathrm{~K}$ is attributed to a high-energy optical magnon mode mixing with low-energy

\footnotetext{
*Corresponding author: s_shamoto@cross.or.jp

Published by the American Physical Society under the terms of the Creative Commons Attribution 4.0 International license. Further distribution of this work must maintain attribution to the author(s) and the published article's title, journal citation, and DOI.
}

acoustic magnon mode and magnon-magnon scattering [10]. It is reported that the energy of the optical mode is lowered by increasing the temperature [11]. The low-lying acoustic mode has the chirality in the magnetic susceptibility $\chi_{x y}^{\prime \prime}$, whereas the optical mode shows the opposite chirality $\chi_{y x}^{\prime \prime}$ [12]. The difference between the two modes with the alternating chiralities produces the spin current. Therefore, the mixture of two chirality modes facilitated by lowering the optical mode and magnon-magnon scattering strongly suppresses the spin current generation [10]. This is the degradation mechanism of the spin Seebeck effect at high temperatures. On the other hand, it should be noted that a strong magnetic field dependence between 0.18 and $8 \mathrm{~T}$ in the spin Seebeck effect appears only below $30 \mathrm{~K}$ [8]. In addition, the large magnetocapacitance effect in YIG is observed below $90 \mathrm{~K}$ [13]. Although YIG is one of the most popular ferrimagnets, the ultralow-energy magnetic excitations have been measured only near room temperature [14-16]. Therefore, the MDOS, $\mathcal{D}_{M}$, at low temperatures measured by inelastic neutron scattering (INS) may provide us new insight into the origins of these ultralow-energy anomalies. The magnon dispersions of YIG have been measured by INS [11,17-19] because INS is one of the best tools to study the magnon dispersion over a broad rang of a momentum-energy $(Q, E)$ space. Furthermore, owing to the recent high-intensity pulsed neutron sources, it becomes possible to study the energy dependence of the magnon dispersions up to $200 \mathrm{meV}$ and also measure them down to the low energies such as $10 \mu \mathrm{eV}$ by using high energy-resolution backscattering spectrometers. In our recent study [18], a universal relation between the MDOS and the $Q$-integrated dynamical spin susceptibility $\chi^{\prime \prime}(E)$ was 
obtained based on the isotropic quadratic dispersion model. Thus, combining this model with the high-energy resolution of DNA, the magnon dispersion at the ultralow energy can be estimated with high accuracy that is sensitive to the energy gap in the magnon dispersion. Here we report an anomalous behavior that the Zeeman gap is closing even under a magnetic field only along [111] at low temperatures revealed by the ultralow-energy measurement of the high-energy-resolution backscattering spectrometer DNA at J-PARC. The tendency is supported by specific heat capacity measurement under the magnetic field. We also observed the peak structure below $30 \mathrm{~K}$ in the temperature dependence of magnetization only under the magnetic field along [111].

\section{EXPERIMENTAL PROCEDURES}

A single crystal of YIG was grown by a traveling solvent floating zone furnace [20] with four halogen lamps (FZT-4000-H-II-S-TS, Crystal Systems Co., Ltd.) at a rate of $0.6 \mathrm{~mm} / \mathrm{h}$ under an air flow of $2 \mathrm{~L} / \mathrm{min}$. The crystal was grown along the magnetic easy axis [111] at an accuracy within $3^{\circ}$ from a seed YIG crystal. The crystal was $5.5 \mathrm{~mm}$ in diameter and $38 \mathrm{~mm}$ in length with a weight of $5.1 \mathrm{~g}$. YIG has a crystallographic cubic symmetry [21] of $I a \overline{3} d$ (no. 230) with a lattice parameter $a=12.38 \AA$ at $300 \mathrm{~K}$. The chemical composition is $\mathrm{Y}_{2.84 \pm 0.09} \mathrm{Fe}_{5} \mathrm{O}_{11.57 \pm 0.21}$, determined by a single-crystal neutron diffraction analysis [18].

A magnetic field of about $0.1 \mathrm{~T}$ was applied along the [111] direction, which is the rod crystal growth direction to remove magnetic domain walls by a pair of permanent magnets, Magfine NR0010 (Aichi Steel Co., Ltd.) with a residual magnetic flux density $B_{r} \sim 1.2 \mathrm{~T}$.

Ultralow-energy magnon excitation of YIG has been measured below $45 \mu \mathrm{eV}$ by an inverted-geometry spectrometer DNA (BL02) [22] at J-PARC. A high-energy resolution of $3.44 \pm 0.02 \mu \mathrm{eV}$ at $E=0 \mathrm{meV}$ was obtained by using pulseshaping chopper with a $3 \mathrm{~cm}$ slit at a rotating speed of $225 \mathrm{~Hz}$. The vertical $Q$ resolution was about $0.02 \AA^{-1}$. UTSUSEMI software is used for the analysis of the data sets obtained at DNA [23]. By normalizing the observed magnon intensity to a standard vanadium tube with a similar size to our YIG crystal, the $Q$-integrated dynamical spin susceptibility $\chi^{\prime \prime}(E)$ was obtained [18]. The magnetic Bragg peak tail was subtracted from the inelastic scattering intensity by a fitted Gaussian peak. Because of the ambiguity of the ultralow-energy magnetic Bragg tail, intensities below $10 \mu \mathrm{eV}(12 \mu \mathrm{eV}$ at $10 \mathrm{~K})$ were excluded. The Bragg tail subtraction was checked by the energy dependence of the obtained dynamical spin susceptibility $\chi^{\prime \prime}(E)$, which is expected to have a square root of energy dependence. The errors are estimated as standard deviations.

At the magnetic Bragg point of $\mathbf{Q}=(0,2,-2)$ in Fig. 1, the magnetic field direction is normal to the scattering vector $\mathbf{Q}$. On the other hand, the outer magnetic field $30 \mathrm{~cm}$ away from the sample position was suppressed down to about $0.1 \mathrm{mT}$ by making a magnetic circuit with an iron or permalloy frame through the crystal to prevent possible neutron detector errors at DNA. Here we define the scattering wave vector $\mathbf{Q}$ as $\mathbf{Q}=Q_{a}(1,1,1)+Q_{b}(0,-1,1)+$ $Q_{c}(2,-1,-1) . Q_{a}, Q_{b}$, and $Q_{c}$ are in reciprocal lattice units (r.l.u.). The final energy $E_{f}$ of DNA with $\operatorname{Si}(111)$ analyzer
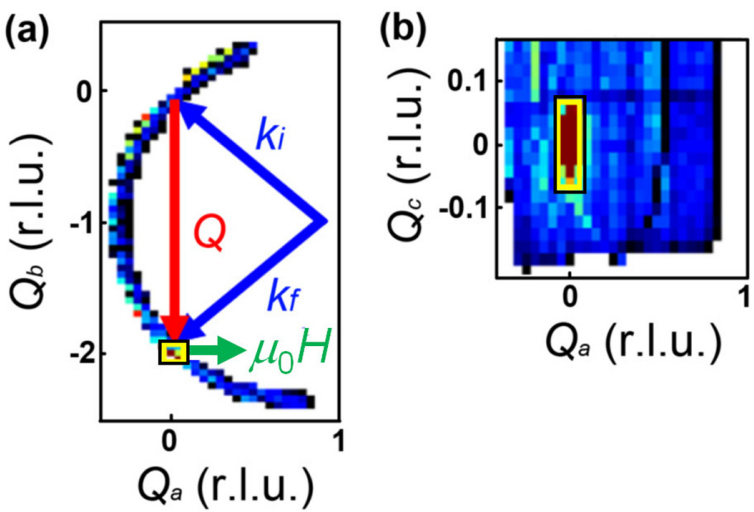

FIG. 1. Crystal setting diagram in $\left(Q_{a}, Q_{b}, Q_{c}\right)$ at DNA spectrometer. (a) $Q_{a}-Q_{b}$ scattering plane. $\mathbf{Q}$ is the scattering vector of $(0,2,-2)$. Magnetic field $\mu_{0} H$ is applied normal to $\mathbf{Q}$. (b) $Q_{a}-Q_{c}$ reciprocal plane. The intensity in the yellow rectangular region is integrated.

was $2.08 \mathrm{meV}$. The intensity around $(0,2,-2)$ was integrated in the range of \pm 0.05 r.l.u. in $Q_{a}, \pm 0.04$ r.l.u. in $Q_{b}$, and \pm 0.075 r.l.u. in $Q_{c}$, as indicated by the rectangular region in Fig. 1 . The $(0,2,-2)$ is the strongest magnetic Bragg peak, which includes the nuclear Bragg reflection with $2.2 \%$ of the total intensity. The INS measurements were carried out at 10 , $50,100,150,200$, and $300 \mathrm{~K}$. It typically takes about $10 \mathrm{~h}$ for one measurement under a proton beam power of about $300 \mathrm{~kW}$ at J-PARC. The neutron absorption coefficient $A^{*}$ of our single-crystal YIG was estimated to be 0.54 based on our numerical calculation. The $Q$-integrated magnon intensity at each energy was obtained by the full integration in 3D reciprocal space at around the $\Gamma$ point. Magnetic susceptibility and specific heat capacity measurements were carried out under magnetic field cooling using single crystals with $\sim 20 \mathrm{mg}$ by Physical Property Measurement System with $9 \mathrm{~T}$ magnet (Quantum Design Co., Ltd.). The applied magnetic fields may be reduced to less than two thirds of the applied values due to the demagnetization effect of our small block crystals. On the other hand, a large single crystal for neutron scattering has a cylindrical shape along the magnetic field, leading to the negligible demagnetization effect. This is proved by the Zeeman energy gap value as described below.

\section{RESULTS AND DISCUSSION}

To estimate the magnon dispersion from the energy dependence of the dynamical spin susceptibility $\chi^{\prime \prime}(E)$ on an absolute scale, we use the following equation:

$$
\begin{aligned}
\left(\frac{d^{2} \sigma}{d \Omega d E}\right)_{M}= & \frac{\left(\gamma r_{\mathrm{e}}\right)^{2}}{\pi g^{2} \mu_{\mathrm{B}}^{2}} \frac{\mathbf{k}_{f}}{\mathbf{k}_{i}} f^{2}(Q) t^{2}(\mathbf{Q})\left\{1+(\hat{\tau} \cdot \hat{\eta})^{2}\right\}_{a v} \\
& \times\{1+n(E)\} \chi^{\prime \prime}(\mathbf{Q}, E),
\end{aligned}
$$

where the constant value $\left(\gamma r_{e}\right)^{2}=0.2905 \mathrm{barn} \mathrm{sr}^{-1}, g$ is the Landé g-factor, $\mu_{\mathrm{B}}$ is the Bohr magneton, $\mathbf{k}_{i}$ and $\mathbf{k}_{f}$ are the incident and final wave vectors, and the isotropic magnetic form factor $f^{2}(Q)$ of $\mathrm{Fe}^{3+}$ at the (220) plane is 0.8059 $\left(Q=1.44 \AA^{-1}\right)$. The dynamic structure factor $t^{2}(\mathbf{Q})[24]$ is approximated to be a squared static magnetic structure factor 
(a)

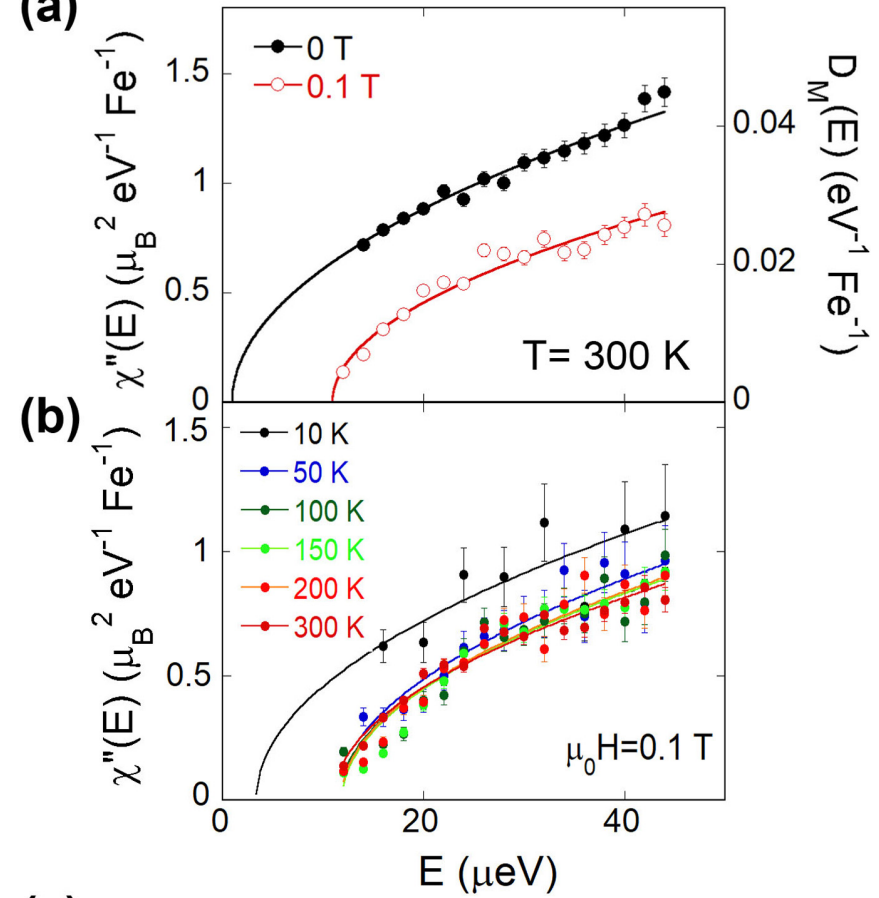

(c)

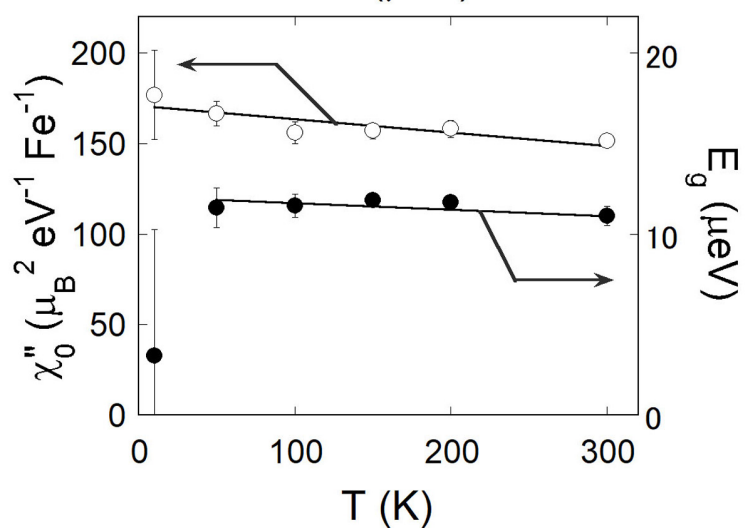

FIG. 2. Energy dependence of $Q$-integrated dynamical spin susceptibilities $\chi^{\prime \prime}(E)$. (a) $\chi^{\prime \prime}(E)$ and $\mathcal{D}_{M}$ at $300 \mathrm{~K}$ with (open circles) and without (closed circles) a magnetic field. Solid lines are fitted by Eq. (2). (b) $\chi^{\prime \prime}(E)$ in the temperature range from 10 to $300 \mathrm{~K}$ with a magnetic field of $0.1 \mathrm{~T}$ along [111]. The highest values with large error bars are measured at $10 \mathrm{~K}$. (c) Temperature dependence of fitted parameters, $\chi_{0}^{\prime \prime}$ (open circles) and $E_{g}$ (closed circles) in Eq. (2). The solid lines are guides to the eye.

relative to full moments, i.e., $t^{2}(\mathbf{Q}) \approx F_{M}^{2}(\mathbf{G}) / F_{M 0}^{2}=13 / 25$ at $\mathbf{G}=(0,2,-2)$, where $\mathbf{G}$ is a magnetic reciprocal lattice vector, $\hat{\tau}$ is a unit vector in the direction of $\mathbf{Q}, \hat{\eta}$ is a unit vector in the mean direction of the spins, the angle-dependent term $\left\{1+(\hat{\tau} \cdot \hat{\eta})^{2}\right\}_{a v}$ is 1 and $4 / 3$, with and without magnetic fields, respectively, and $n(E)$ is the Bose factor.

The obtained imaginary part of $Q$-integrated dynamical spin susceptibility $\chi^{\prime \prime}(E)$ in the Brillouin zone is shown as a function of energy in Figs. 2(a) and 2(b). The $E$ dependence of $\chi^{\prime \prime}(E)$ for a quadratic dispersion case becomes a square-root function of energy [8]. Based on our previous study [18], the MDOS in the quadratic dispersion model is proportional to $\chi^{\prime \prime}(E)$. The dynamical spin susceptibility $\chi^{\prime \prime}(E)$ is described as follows:

$$
\chi^{\prime \prime}(E)=\chi_{0}^{\prime \prime} \sqrt{E-E_{g}},
$$

where the coefficient is given by $\chi_{0}^{\prime \prime}=\left\{g^{2} \mu_{\mathrm{B}}^{2} S(S+\right.$ 1) $\left.D^{-3 / 2}\right\} /\left\{(2 \pi)^{2} 40\right\}$ with a stiffness constant $D, 40$ the number of $\mathrm{Fe}$ sites in the crystal unit cell, and the spin $S=5 / 2$. The value $g^{2} \mu_{\mathrm{B}}^{2} S(S+1)$ is about $35 \mu_{\mathrm{B}}^{2} \mathrm{Fe}^{-1}$ for $\mathrm{Fe}^{3+}$. The energy gap $E_{g}$ in YIG is approximated as the summation of Zeeman energy by an applied magnetic field $\mu_{0} H$ and anisotropy field $K S_{z}^{2}$ as follows:

$$
E_{g}=g \mu_{\mathrm{B}} \mu_{0} H+K S_{z}^{2},
$$

where the latter anisotropy term is estimated as $0.9 \pm 0.5 \mu \mathrm{eV}$ from a zero-field measurement. The total gap value becomes $11.0 \pm 0.5 \mu \mathrm{eV}$ from a magnetic field measurement. The Zeeman energy gap becomes $10.1 \pm 0.7 \mu \mathrm{eV}$, suggesting the applied magnetic field $\mu_{0} H$ of $0.088 \pm 0.006 \mathrm{~T}$. The obtained value is consistent with $0.1 \pm 0.01 \mathrm{~T}$ measured by a Gauss meter 5180 (F. W. Bell Co., Ltd.). The magnetic intensity is expected to decrease under a magnetic field normal to the $Q$ vector by a factor of $3 / 4$. The solid lines with the fixed ratio reproduce the intensities very well as shown in Fig. 2(a). Based on this result, we can conclude that the magnetic domains are randomly oriented under no magnetic field, whereas the magnetic domain walls are fully removed from the crystal under the magnetic field of $0.1 \mathrm{~T}$. Figure 2(b) shows the temperature dependence of the MDOS. It abruptly increases at $T=10 \mathrm{~K}$. The fitted parameters are shown in Fig. 2(c). The Zeeman energy gap appreciably closed at $10 \mathrm{~K}$. The observed intensity at $10 \mathrm{~K}$ was weak due to the small Bose factor, resulting in the scattering of $\chi^{\prime \prime}(E)$ in Fig. 2(b). In the fitting, ambiguity remains due to the large errors. However, the closed gap at $10 \mathrm{~K}$ is confirmed by the specific heat capacity measurement under the magnetic field along [111] as discussed later. Based on the energy dependence of $\chi^{\prime \prime}(E)$, the magnon stiffness constant $D$ is estimated by

$$
D=\left\{\frac{g^{2} \mu_{\mathrm{B}}^{2} S(S+1)}{(2 \pi)^{2} 40 \chi_{0}^{\prime \prime}}\right\}^{2 / 3} \text {. }
$$

Then, by using the stiffness constant $D$, the magnon dispersion becomes

$$
E=D a^{2} q^{2}+E_{g} .
$$

In Fig. 2(c) $\chi_{0}^{\prime \prime}$ increases from $151.6 \pm 3.7 \mu_{\mathrm{B}}{ }^{2} \mathrm{eV}^{-3 / 2}$ $\mathrm{Fe}^{-1}$ at $T=300 \mathrm{~K}$ to $177 \pm 25 \mu_{\mathrm{B}}^{2} \mathrm{eV}^{-3 / 2} \mathrm{Fe}^{-1}$ at $10 \mathrm{~K}$. The temperature dependence of $\chi_{0}^{\prime \prime}$ suggests the slight softening of magnon with decreasing temperature, contrary to our expectations. The stiffness constant $D a^{2}$ estimated from $\chi_{0}^{\prime \prime}$ decreases from $425 \pm 7 \mathrm{meV} \AA^{2}$ at $300 \mathrm{~K}$ to $383 \pm 76 \mathrm{meV} \AA^{2}$ at $10 \mathrm{~K}$. The value at $10 \mathrm{~K}$ is smaller than our previous result of $633 \pm 17 \mathrm{meV} \AA^{2}$ at $\sim 20 \mathrm{~K}$ obtained from the magnon dispersion in an energy range from 2 to $14 \mathrm{meV}$ [18]. These results suggest the suppression of Zeeman energy gap and magnon softening at $10 \mathrm{~K}$ under the magnetic field along [111].

To confirm the magnetic anomaly below $50 \mathrm{~K}$, the magnetization and specific heat capacity of YIG have been measured. They also give us important information about the low-energy magnons [25]. Figures 3(a) and 3(b) show the temperature 

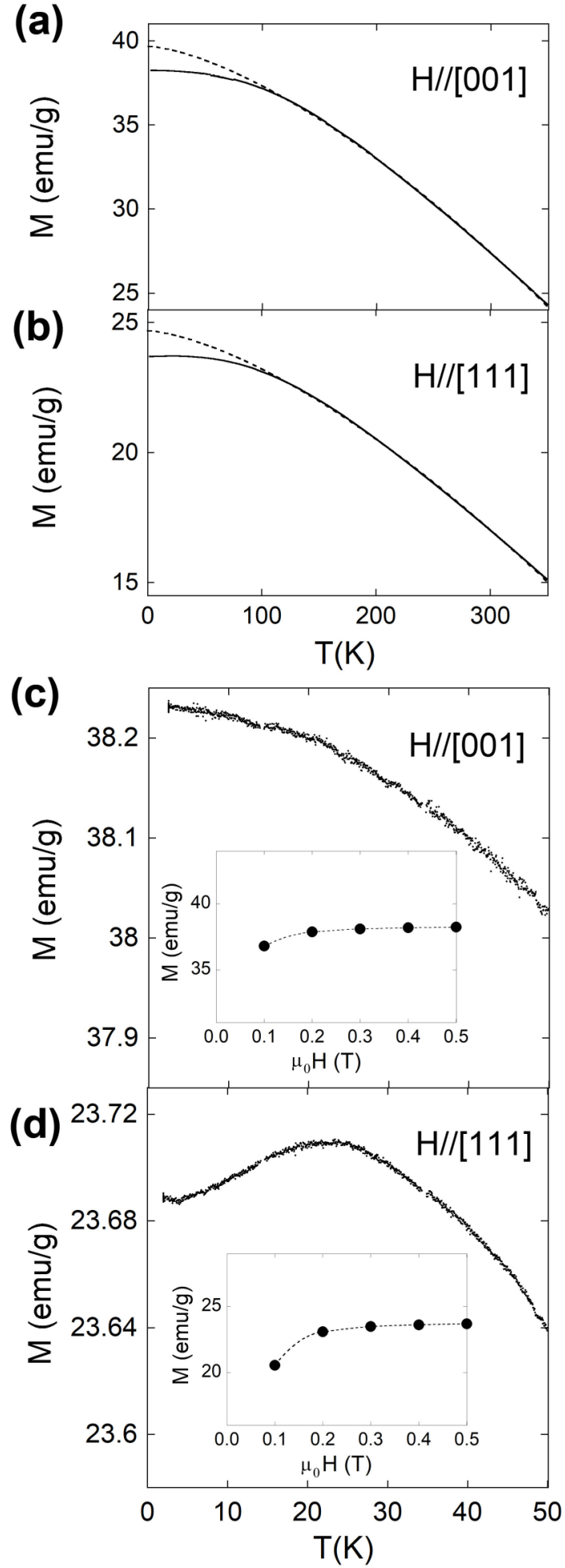

FIG. 3. Magnetizations as a function of temperature. Observed magnetization under a magnetic field of $0.5 \mathrm{~T}$ along [001] (a) and [111] (b) (solid lines) with Bloch-type magnetizations (broken lines) fitted in the temperature range from 150 to $350 \mathrm{~K}$. Enlarged temperature dependences of magnetizations at $\mu_{0} H=0.5 \mathrm{~T}$ along [001] (c) and [111] (d). The insets show the magnetic field dependence of the magnetizations. The horizontal thin line in (d) is a guide to the eye. dependence of magnetization under the magnetic field cooling of $0.5 \mathrm{~T}$ along [001] and [111], respectively. Because there is ambiguity of the demagnetization effect of the measured small crystals, the magnetic field of $0.5 \mathrm{~T}$ was applied in Fig. 3. The insets in Figs. 3(c) and 3(d) show the magnetic field dependence from 0.1 to $0.5 \mathrm{~T}$. They show that $0.1 \mathrm{~T}$ is not large enough to have the full magnetization of YIG due to the demagnetization effect of the small crystals. At low temperatures, the magnetization is usually decreased by the magnon excitation, where the temperature dependence is expressed by the Bloch $T^{3 / 2}$ rule [8]. However, the Bloch rule can be applied to the magnetization only above $150 \mathrm{~K}$ as shown in Figs. 3(a) and 3(b). The fittings lead to similar values of $\zeta=5.96$ to $5.97 \times 10^{-5} \mathrm{~K}^{-3 / 2}$ in $M=M_{0}\left(1-\zeta T^{3 / 2}\right)$. They are slightly larger than the reported values, 5.20 to $5.83 \times 10^{-5} \mathrm{~K}^{-3 / 2}$, in the previous study [8]. Below $50 \mathrm{~K}$, the magnetization under a magnetic field along [001] shows a continuous increase with decreasing temperature [Fig. 3(c)]. On the other hand, a peak appears at approximately $25 \mathrm{~K}$ in the magnetic field along [111] [Fig. 3(d)]. A plausible explanation for the peak is that the onset of a canted ferrimagnet in a magnetic field along [111].

Specific heat capacity measurement has also been carried out to observe the anomalous behavior at around $25 \mathrm{~K}$ as shown in Figs. 4(a) and 4(b). A three-dimensional ferromagnet or ferrimagnet magnon without a gap is expected to show a specific heat capacity proportional to $T^{3 / 2}$, in addition to the phonon term of $T^{3}$ at low temperature [26] as follows:

$$
\frac{C}{T^{3 / 2}}=A+B T^{3 / 2},
$$

where $\quad A=0.113 k_{B}\left(D a^{2} / k_{B}\right)^{-3 / 2}, \quad B=12 \pi^{4} N_{A} k_{B} /\left(5 \theta_{D}^{3}\right)$ with $k_{B}$ the Boltzmann constant, $N_{A}$ the Avogadro number for the formula weight $\left(\mathrm{Y}_{3} \mathrm{Fe}_{5} \mathrm{O}_{12}\right)$, and $\theta_{D}$ the Debye temperature. In Figs. 4(a) and 4(b), $C / T^{3 / 2}$ is plotted as a function of $T^{3 / 2}$. Figure 4(a) shows the specific heat capacity in a wide temperature range. The difference between two magnetic field directions is apparent below around $150 \mathrm{~K}$. It suggests that the magnon anomaly starts from $150 \mathrm{~K}$, as observed in the non-Bloch-type temperature dependence of magnetization below $150 \mathrm{~K}$. In a ferromagnetic resonance study of YIG, the first-order anisotropy constant shows an anomalous increase below $150 \mathrm{~K}$ [27]. They may have a common origin, although the anomaly was not detected by the present neutron scattering study. Figure 4(b) shows the same data at low temperatures below $14 \mathrm{~K}$. Extrapolation to the $y$ axis gives the stiffness constant $D a^{2}$, whereas the slope is related to the Debye temperature $\theta_{D}$, which is expected to be common for all the plots in Fig. 4(b). However, both parameters below $9 \mathrm{~K}\left(\sim 25 K^{3 / 2}\right)$ are very different between the two plots. In the case of $\mu_{0} H$ along [001], $A$ becomes $-2.8 \pm 0.2 \times 10^{-4} \mathrm{~J} / \mathrm{mole} / \mathrm{K}^{5 / 2}$, whereas $A$ is $8.17 \pm$ $0.02 \times 10^{-3} \mathrm{~J} / \mathrm{mole} / \mathrm{K}^{5 / 2}$ at $\mu_{0} H$ along [111]. This finite magnon contribution to the specific heat capacity suggests the disappearance of the energy gap in the magnon dispersion. The stiffness constant $D a^{2}$ from the $A$ value at the magnetic field along [111] was estimated as $312 \mathrm{meV} \AA^{2}$. This value is 

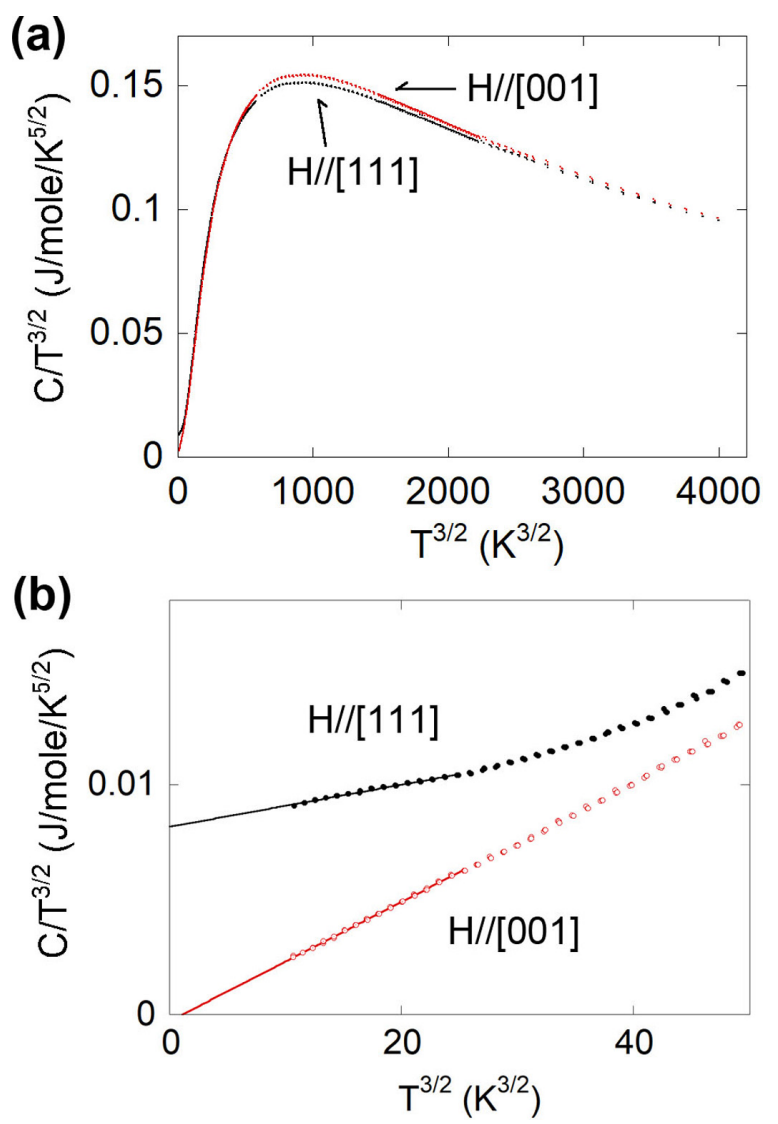

FIG. 4. Specific heat capacity $C / T^{3 / 2}$ is plotted as a function of $T^{3 / 2}$. (a) The magnetic field directions are parallel to [001] (red dots) and [111] (black dots) under $1 \mathrm{~T}$. Both heat capacities are plotted in a wide temperature range up to $250 \mathrm{~K}$. The upper values (red dots) around a peak at $T=96 \mathrm{~K}\left(\sim 920 \mathrm{~K}^{3 / 2}\right)$ are measured at $\mu_{0} H$ $=1 \mathrm{~T}$ along [001], which become lower at low temperatures than those (black dots) measured at $\mu_{0} H=1 \mathrm{~T}$ along [111]. (b) Enlarged temperature dependence of the heat capacities for [001] (red open circles) and [111] (black closed circles) magnetic fields at low temperature below $14 \mathrm{~K}$. The difference becomes large below $T=$ $9 \mathrm{~K}\left(\sim 25 \mathrm{~K}^{3 / 2}\right)$. The linear solid lines are fitted results within the range, extrapolated to the ends.

consistent with $383 \pm 76 \mathrm{meV} \AA^{2}$ obtained from an ultralowenergy magnon at $10 \mathrm{~K}$. These values are smaller than our previous result of $633 \pm 17 \mathrm{meV} \AA^{2}$ estimated from the highenergy magnon at $\sim 20 \mathrm{~K}$. The consistency of two stiffness constants obtained from two methods at low temperature suggests low-energy dispersion flattening from the simple quadratic dispersion in addition to the disappearance of the Zeeman energy gap. As for the Debye temperature, it changed the value depending on the magnetic field direction. In the case of a magnetic field along [100], the Debye temperature was $195.8 \mathrm{~K}$, whereas it became $277.0 \mathrm{~K}$ at the magnetic field along [111]. This lattice hardening is observed below $9 \mathrm{~K}$ as shown in Fig. 4(b). In the whole range of temperature, there were no peaks in the temperature dependence of specific heat capacity, suggesting no appreciable phase transition. Therefore, this anomaly can be categorized into a crossover from a ferrimagnet to a canted ferrimagnet.
Let us discuss the new crossover observed at around $25 \mathrm{~K}$ below the precursor anomaly at around $150 \mathrm{~K}$. As for the precursor anomaly, the deviation from Bloch rule below $150 \mathrm{~K}$ in Fig. 3(a) reaches at 3.6\%(H $\|[100])$ and $4.0 \%(\mathbf{H} \|[111])$ at the lowest temperature of $2.5 \mathrm{~K}$. The amount of suppressions corresponds to about $15.4^{\circ}$ and $16.3^{\circ}$ in the canting angles of magnetic moments, respectively. On the other hand, the observed magnetization suppression by the peak indicated in Fig. 3(d) is the order of $0.1 \%$. Although it is small, this magnetization anomaly accompanies the Zeeman-gap closure. The origin of this anomaly is expected to be the same as the dielectric relaxation anomaly identified in the same temperature and the same magnetic field direction dependences [13]. For the appearance of dielectric constants in this temperature range, the lattice distortion should couple to the spin via a spin-orbit coupling. As for the crystal structure of YIG, it is trigonally distorted at room temperature [18]. Therefore, the symmetry axis [111] coincides with the present magnetic field direction along the [111] axis, which is also the magnetic easy axis. However, it is puzzling why the dielectric property appears at the low temperatures without any phase transition. One possible scenario is that thermally activated itinerant $\mathrm{Fe}^{2+}$ impurities are frozen out at the low temperature [13]. Impurity $\mathrm{Fe}^{2+}$ centers are expected to provide large spin-orbit coupling effects [28]. Therefore, the observed magnetic anomaly may appear through the spin-orbit coupling effect due to the localization of $\mathrm{Fe}^{2+}$ centers. Since this perspective is worth further investigating we will pursue this subject in a future study.

Low-temperature spin Seebeck effect strongly depends on the ultralow-energy MDOS. The present anomaly appears under a magnetic field along [111]. So far, there is no experiment under this condition. It is necessary to check how the spin Seebeck effect changes depending on the magnetic field directions relative to the crystal axis. Closing the Zeeman energy gap must increase the spin current at low temperatures. On the other hand, the $\chi_{x y}^{\prime \prime}$ chirality mode may change the character with the spin canting in the low-lying acoustic magnon mode, which might effectively reduce the spin current. These types of experiments may provide further information about the spin Seebeck effect.

\section{CONCLUSIONS}

The dynamical spin susceptibility $\chi^{\prime \prime}(E)$ is measured on an absolute scale at the high-energy resolution backscattering spectrometer DNA of J-PARC MLF as a function of energy in the ultralow-energy range below $45 \mu \mathrm{eV}$. By using the relation between the magnon dispersion and the dynamical spin susceptibility $\chi^{\prime \prime}(E)$, it is possible to investigate ultralowenergy magnons. This method is effective especially for the study of ultralow-energy magnons. By this method, the Zeeman energy gap anomaly is found under a magnetic field of about $0.1 \mathrm{~T}$ along [111] below $50 \mathrm{~K}$ in YIG. The similar anomaly is also observed by the magnetization measurement under the magnetic field along the same direction. In addition, the specific heat capacity measurement also reveals that the Zeeman energy gap closes at low temperatures. The present results suggest that the ultralow-energy magnon dispersion is flattened from the simple quadratic function under the magnetic field. All these anomalies correspond to our find- 
ing of a magnetic crossover in YIG below $30 \mathrm{~K}$ under the magnetic field along [111]. This anomaly can be used to reveal spintronic phenomena, such as spin Seebeck and ultrasound spin pumping effects.

\section{ACKNOWLEDGMENTS}

This work at J-PARC has been performed at DNA(BL02) under the Proposals No. 2017L0301, No. 2014B0157, No.
2015I0002, and No. 2016A0318. We acknowledge Dr. K. Shibata, Dr. H. Onishi, Dr. T. Kikkawa, Dr. N. Sato, Dr. R. Kajimoto, Dr. M. Mori, Prof. Y. Nambu, Prof. S. E. Barnes, and Prof. S. Maekawa for discussions, the CROSS sample environment team for experimental assistance, and $\mathrm{M}$. Usami in the JAEA technical support team for making the sample holders. This work was supported by the Iketani Science and Technology Foundation 0311086-A (JP) and JSPS KAKENHI Grants No. JP25287094 and No. JP16K05424.
[1] Y. Kajiwara, K. Harii, S. Takahashi, J. Ohe, K. Uchida, M. Mizuguchi, H. Umezawa, H. Kawai, K. Ando, K. Takanashi, S. Maekawa, and E. Saitoh, Transmission of electrical signals by spin-wave interconversion in a magnetic insulator, Nature (London) 464, 262 (2010).

[2] K. Uchida, J. Xiao, H. Adachi, J. Ohe, S. Takahashi, J. Ieda, T. Ota, Y. Kajiwara, H. Umezawa, H. Kawai, G. E. W. Bauer, S. Maekawa, and E. Saitoh, Spin Seebeck insulator, Nat. Mater. 9, 894 (2010).

[3] K. Uchida, H. Adachi, T. Ota, H. Nakayama, S. Maekawa, and E. Saitoh, Observation of longitudinal spin-Seebeck effect in magnetic insulators, Appl. Phys. Lett. 97, 172505 (2010).

[4] K. Uchida, H. Adachi, T. An, T. Ota, M. Toda, B. Hillebrands, S. Maekawa, and E. Saitoh, Long-range spin Seebeck effect and acoustic spin pumping, Nat. Mater. 10, 737 (2011).

[5] A. A. Serga, A. V. Chumak, and B. Hillebrands, YIG magnonics, J. Phys. D: Appl. Phys. 43, 264002 (2010).

[6] A. V. Chumak, V. I. Vasyuchka, A. A. Serga, and B. Hillebrands, Magnon spintronics, Nat. Phys. 11, 453 (2015).

[7] C. Hauser, T. Richter, N. Homonnay, C. Eisenschmidt, M. Qaid, H. Deniz, D. Hesse, M. Sawicki, S. G. Ebbinghaus, and G. Schmidt, Yttrium iron garnet thin films with very low damping obtained by recrystallization of amorphous material, Sci. Rep. 6, 20827 (2016).

[8] T. Kikkawa, K. Uchida, S. Daimon, Z. Qiu, Y. Shiomi, and E. Saitoh, Critical suppression of spin Seebeck effect by magnetic fields, Phys. Rev. B 92, 064413 (2015).

[9] H. Jin, S. R. Boona, Z. Yang, R. C. Myers, and J. P. Heremans, Effect of the magnon dispersion on the longitudinal spin Seebeck effect in yttrium iron garnets, Phys. Rev. B 92, 054436 (2015).

[10] J. Barker and G. E. W. Bauer, Thermal Spin Dynamics of Yttrium Iron Garnet, Phys. Rev. Lett. 117, 217201 (2016).

[11] J. S. Plant, Spinwave dispersion curves for yttrium iron garnet, J. Phys. C: Solid State Phys. 10, 4805 (1977).

[12] Y. Nambu, J. Barker, Y. Okino, T. Kikkawa, Y. Shiomi, M. Enderle, T. Weber, B. Winn, M. Graves-Brook, J.-M. Tranquada, T. Ziman, M. Fujita, G.E.W. Bauer, E. Saitoh, and K. Kakurai, Observation of Magnon Polarization, Phys. Rev. Lett. 125, 027201 (2020).

[13] Y. Yamasaki, Y. Kohara, and Y. Tokura, Quantum magnetoelectric effect in iron garnet, Phys. Rev. B 80, 140412(R) (2009).

[14] S. O. Demokritov, B. Hillebrands, and A. N. Slavin, Brillouin light scattering studies of confined spin waves: Linear and nonlinear confinement, Phys. Rep. 348, 441 (2001).
[15] K. S. Olsson, K. An, X. Ma, S. Sullivan, V. Venu, M. Tsoi, J. Zhou, L. Shi, and X. Li, Temperature-dependent Brillouin light scattering spectra of magnons in yttrium iron garnet and permalloy, Phys. Rev. B 96, 024448 (2017).

[16] Y. Hashimoto, S. Daimon, R. Iguchi, Y. Oikawa, K. Shen, K. Sato, D. Bossini, Y. Tabuchi, T. Satoh, B. Hillebrands, G. E. W. Bauer, T. H. Johansen, A. Kirilyuk, T. Rasing, and E. Saitoh, All-optical observation and reconstruction of spin wave dispersion, Nat. Commun. 8, 15859 (2017).

[17] J. S. Plant, 'Pseudo-acoustic' magnon dispersion in yttrium iron garnet, J. Phys. C: Solid State Phys. 16, 7037 (1983).

[18] S. Shamoto, T. U. Ito, H. Onishi, H. Yamauchi, Y. Inamura, M. Matsuura, M. Akatsu, K. Kodama, A. Nakao, T. Moyoshi, K. Munakata, T. Ohhara, M. Nakamura, S. Ohira-Kawamura, Y. Nemoto, and K. Shibata, Neutron scattering study of yttrium iron garnet, Phys. Rev. B 97, 054429 (2018).

[19] A. J. Princep, R. A. Ewings, S. Ward, S. Toth, C. Dubs, D. Prabhakaran, and A. T. Boothroyd, The full magnon spectrum of yttrium iron garnet, npj Quantum Mater. 2, 63 (2017).

[20] S. Kimura and I. Shindo, Single crystal growth of YIG by the floating zone method, J. Cryst. Growth 41, 192 (1977).

[21] D. Rodic, M. Mitric, R. Tellgren, H. Rundlof, and A. Kremenovic, True magnetic structure of the ferrimagnetic garnet $\mathrm{Y}_{3} \mathrm{Fe}_{5} \mathrm{O}_{12}$ and magnetic moments of iron ions, J. Magn. Magn. Mater. 191, 137 (1999).

[22] K. Shibata, N. Takahashi, Y. Kawakita, M. Matsuura, T. Yamada, T. Tominaga, W. Kambara, M. Kobayashi, Y. Inamura, T. Nakatani, K. Nakajima, and M. Arai, The performance of TOF near backscattering spectrometer DNA in MLF, J-PARC, JPS Conf. Proc. 8, 036022 (2015).

[23] Y. Inamura, T. Nakatani, J. Suzuki, and T. Otomo, Development status of software "Utsusemi" for chopper spectrometers at MLF, J-PARC, J. Phys. Soc. Jpn. 82, SA031 (2013).

[24] G. Shirane, S. M. Shapiro, and J. M. Tranquada, Neutron Scattering with a Triple-Axis Spectrometer (Cambridge University Press, Cambridge, 2002).

[25] J. Barker and G. E. W. Bauer, Semiquantum thermodynamics of complex ferrimagnets, Phys. Rev. B 100, 140401(R) (2019).

[26] C. Kittel, Quantum Theory of Solids (John Wiley \& Sons, New York, 1987).

[27] J. F. Dillon, Jr., Ferrimagnetic resonance in yttrium iron garnet, Phys. Rev. 105, 759 (1957).

[28] Y. Kohara, Y. Yamasaki, Y. Onose, and Y. Tokura, Excesselectron induced polarization and magnetoelectric effect in yttrium iron garnet, Phys. Rev. B 82, 104419 (2010). 